\title{
Analysis on restoring stiffness and its hysteresis behavior of slender catenary mooring-line
}

\author{
Yilun Li ${ }^{\text {a,e,1 }}$, Shuangxi Guo ${ }^{\text {b,c,d,1, Weimin Chen }}{ }^{\text {b,d,* }}$, Dingbang Yan ${ }^{\text {b,d }}$, Jixiang Song ${ }^{\text {b,d }}$ \\ ${ }^{\text {a }}$ Lab. MSSMat, CentraleSupélec, Université de Paris-Saclay, Gif sur Yvette, 91190, France \\ ${ }^{\mathrm{b}}$ Key Laboratory of Mechanics in Fluid Solid Coupling System, Institute of Mechanics, Chinese Academy of Sciences, Beijing, 100190, China \\ ${ }^{c}$ AVIC Manufacturing Technology Institute, AVIC Composite Technology Center, Beijing, 101300, China \\ ${ }^{\mathrm{d}}$ School of Engineering Science, University of Chinese Academy of Sciences, Beijing, 100049, China \\ ${ }^{\mathrm{e}}$ Sino-French Engineering School, Beijing University of Aeronautics and Astronautics, Beijing, 100191, China
}

\section{A R T I C L E I N F O}

\section{Keywords:}

Dynamic response

Damping

Hysteresis behavior

Restoring performance

\begin{abstract}
A B S T R A C T
In deeper ocean area, more and more floating oil/gas production platforms or wind turbine have been in operation instead of the fixed truss in shallow water. These floaters need to be located among a range of position by its mooring system, e.g. tension tendon and catenary mooring-line, so as to guarantee structural safety and stability under environmental loads. Catenary mooring-line is increasingly used due to its lower cost and easier installation. Its dynamics coming from structure and fluid flow becomes more obvious with the increase of structural length, which may introduce significant impact on restoring performance of mooring-line. In this study, the non-linearly restoring performance and the hysteresis behavior of mooring-line's stiffness are studied. The nonlinear dynamic model of the mooring-line is established based on the improved FEM simulations. It is found that the restoring stiffness of the catenary mooring-line presents a hysteresis characteristic, principally owing to the damping effect of the catenary dynamics, which gets more obvious with the increase of motion amplitude/frequency. And, the mooring-line dynamics has significant impacts on the tension (related to its restoring stiffness) and displacement response, e.g. the maximum tension amplitude is up to 5.2 times of the quasi-static value if slack happens.
\end{abstract}

\section{Introduction}

As we know, more and more oil, gas, wind and mine industries have been developing towards ocean area because of huge volumes of energy resources in ocean. Instead of the fixed structures onshore, offshore structures are usually floating bodies, such as TLP, Spar and submarine rig/production platform and wind turbine, which need to be fixed or controlled at expected position by its supporting system. Generally, according to the types of floaters and water depth, these mooring systems have mainly three categories (Musial et al., 2004), i.e. catenary mooring-lines, tendon lines and vertical tension legs. Catenary (Barrera et al., 2019) and taut mooring line (Xiong et al., 2016) are widely used due to their lower cost and easier installation. For catenary, as the structural length becomes larger, consequently, the dynamic behaviors such as the structural inertia and hydrodynamic damping of the mooring-line become more obvious.

Suffering from environmental loads coming from wind, ocean current and wave, offshore floating system, moored by mooring-lines, often present large-amplitude motion which is one of big concerns to structural stability and safety. By now, there have been fruitful researches (Yong-Pyo et al., 2005; Sarkar and Eatock Taylor, 2002; Mavrakos and Chatjigeorgiou, 1997; Papazoglou et al., 1990; Yong-Pyo et al., 2005; Fan et al., 2017) on catenary mooring-line along with its influence on dynamic response of a floating system, which mostly focus on mooring-line's restoring force (i.e. the top tension) and stability of floating body. To model the top tension, the quasi-static method was originally used, where the underwater mooring-line is simplified as a static spring to support the up-end and consequently, the restoring force is essentially static. For examples, the mooring-line tensions and motions in time and frequency domain were studied (Mavrakos and Chatjigeorgiou, 1997),

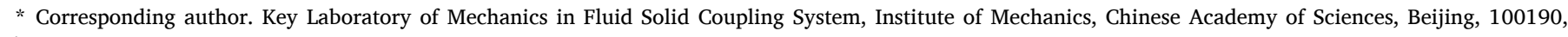
China.

E-mail address: wmchen@imech.ac.cn (W. Chen).

1 These authors contributed equally to this work.
} 
and the parameters predominantly determining the tension of a catenary mooring-line were given (Papazoglou et al., 1990). Yoji Ogawa (1984) gave the equations of static mooring line equilibrium under consideration of gravity and elongation and carried out the static equilibrium characteristics. His results showed that the line elongation cannot be neglected in deep sea. Fan et al. (2017) predicted the mooring line damping for designing truncated mooring system with an improved quasi-static method. Based on the empirical model, the catenary mooring tension caused by vertical motion of the top-end float was presented by Gobat and Grosenbaugh (2001).

However, it was noted that the mooring-line dynamics, coming from the inertia and damping effects of the structure itself and the ambient fluid, becomes more obvious due to the increasingly large values of water depth and catenary span length. This dynamic behavior could lead to a significant impacts on mooring-line tension and, even in some times, introduce a sharp increase of top tension when slack happens. Vassalos and Huang (1996) derived the non-linear equation of a motion cable that subjected to horizontal excitation, and a numerical approach is presented to predict the snap loading of marine cables operating in alternating taut-slack conditions (Huang and Vassalos, 1993). Considering first order geometric nonlinearities, Mansour et al. (2018) obtained exact expression of the catenary curvature and defined the catenary effect on the cable motion; Guo et al. (2016) presented the influences of the amplitude and frequency of SFT motion on mooring line's displacement and dynamic tension, in the numerical model the geometric nonlinearities and the nonlinear fluid force are included. Chen et al. (2001) investigated the dynamic coupling effects between spar float and its mooring lines using the coupled dynamic approach, and the results showed that the tension in mooring lines may greatly increase in the wave frequency range when dynamic forces in mooring lines are considered. Zhang et al. (2012) found that the taut-slack condition significantly amplifies the maximum tension amplitude, the maximum tension may get 5 times larger. Based on laboratory experiments, Hsu et al. (2017) found that the largest values of snap-induced tension may be 1.6 times of the original tension due to involvement of mooring-line dynamics.

The results of top tension of mooring line indicate that its value may increase significantly. In fact, the change of top tension could also have an impact on the restoring stiffness of the mooring system. By now, there is few results on the characteristics and mechanism of dynamic mooringline's restoring stiffness. In this paper, the hysteresis behavior and mechanism of the restoring stiffness are studied, and the displacement and tension response of the catenary mooring line are examined based on the improved finite element approach. In section 2, the dynamic catenary model is established based on the improved FEM method so as to efficiently carry out calculation of nonlinear dynamic response, and the dynamic effects coming from structural dynamics and hydrodynamics are included. Based on our numerical simulations, in section 3 the dynamic response of the catenary under top-end motion, i.e. the restoring stiffness along with displacement and tension, are analyzed and compared with the quasi-static results. Particularly, the restoring performance and its hysteresis are discussed.

\section{Dynamic catenary model and verification}

\subsection{Basic formulas and the improved FEM model of dynamic catenary}

The static tension and restoring force is only related to the configuration and gravity of the structure, and can be obtained with the following equations (Mansour et al., 2018):

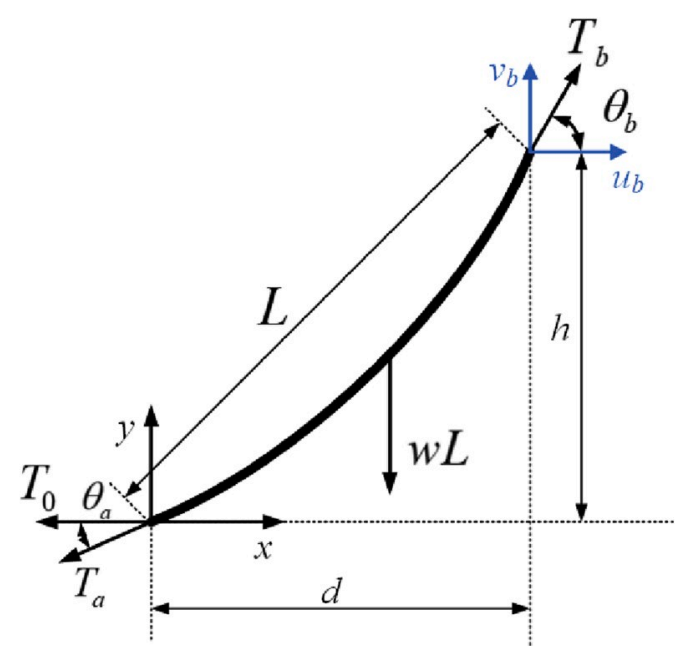

(a) The traditional static force balance of a catenary

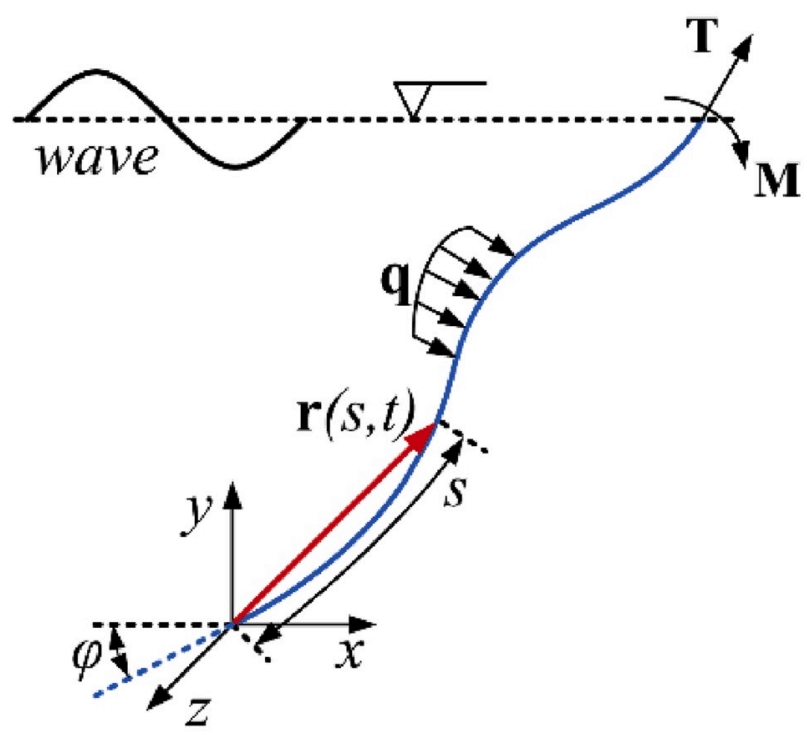

(b) The schematic diagram of a $3 \mathrm{~d}$ flexible mooring-line

Fig. 1. Catenary mooring-line models.

$$
\begin{gathered}
d=\frac{T_{0}}{w}\left[\sinh ^{-1}\left(\tan \theta_{b}\right)-\sinh ^{-1}\left(\tan \theta_{a}\right)\right] \\
h=\frac{T_{0}}{w}\left(\frac{1}{\cos \theta_{b}}-\frac{1}{\cos \theta_{a}}\right) \\
L=\frac{T_{0}}{w}\left(\tan \theta_{b}-\tan \theta_{a}\right)
\end{gathered}
$$

where $d$ is the horizontal projection length, $h$ is the vertical projection length, $L$ is the total length of the catenary, $w$ is the weight per unit length, $T_{0}$ is the horizontal tension at the bottom end, $\theta_{a}$ and $\theta_{b}$ are the angles between the structure and the horizontal axis at the bottom and top end. In this case, only the component caused by structural gravity is considered.

To involve the mooring-line dynamics, there are two dominating models, i.e., the flexible-bar model and lumped mass model, have been developed. In the lumped mass model, mooring line is spatially discretized into several lumped mass nodes which are connected by massless springs (Xiong et al., 2016). Azcona et al. (2017) studied the snap tension and maximum tension of a catenary model using the lumped-mass method and compared his results with the experiments. In the flexible-bar model, globally based position vector and its derivative 
are used to allow an accurate non-linear description of the mooring kinematics. Using the reduced nonlinear motion equations of a cable, Zhang et al. (2010) calculated the displacement, stress and strain of mooring line in deep water, and presented the effect of drag force on the dynamic tension.

Here, instead, the dynamic governing equations in terms of vector equations (Love, 2013; Garrett, 1982) are introduced to consider the dynamics, coming from structural inertial and damping and the fluid drag forces, along with the nonlinear geometry and large displacement. Then, the flexible beam method is applied to obtain the dynamic restoring force of the catenary mooring-line (Lindahl and Sjoberg, 1983; Paulling and Webster, 1986; Kwan and Bruen, 1991; Chen, 2002). The dynamic equations of the catenary (see Fig. 1b) can be written as:

$\mathbf{T}^{\prime}+\mathbf{q}^{\prime}=\rho A \ddot{\mathbf{r}}$

$\mathbf{Q}^{\prime}+\mathbf{r}^{\prime} \times \mathbf{T}+\mathbf{m}=0$

where $\mathbf{T}$ and $\mathbf{Q}$ are respectively the total force and moment of the catenary. $\mathbf{q}$ and $\mathbf{m}$ are respectively the outer force and moment acted on per unit length of the catenary. $\rho$ and $A$ are structural mass density and area respectively. $\mathbf{r}$ represents the position vector. If neglecting the external torque and moment, the governing equation can be rewritten as:

$-\left(E I r^{\prime \prime}\right)^{\prime \prime}+\left(\lambda r^{\prime}\right)^{\prime}+q=\rho A \ddot{r} r^{\prime} \cdot r^{\prime}=(1+\varepsilon)^{2}$

where $\varepsilon$ is the strain of the catenary, $\lambda$ is the effective tension and $E I$ is the bending stiffness. Further, if the bending stiffness in Eq. (4) is zero, we can get the governing equation of catenary mooring-line, which include the gravity force and hydrodynamic load.

As the length of the mooring-line is much larger than its diameter, the Morison equation (Sarkar and Eatock Taylor, 2002; Chen et al., 2001) is employed here to calculate the hydrodynamic force as follow:

$f=\frac{1}{2} C_{d} \rho_{1} D|\mathbf{V}-\dot{\mathbf{r}}|(\mathbf{V}-\dot{\mathbf{r}})+C_{A} \frac{\pi D^{2}}{4} \rho_{1}(\dot{\mathbf{V}}-\ddot{\mathbf{r}})+\frac{\pi D^{2}}{4} \rho_{1} \dot{\mathbf{V}}$

where $D$ and $\mathbf{V}$ are the structural diameter and the vector of the external fluid velocity, $\rho_{1}$ is the density of the fluid, $C_{d}$ and $C_{A}$ are the drag coefficient and added mass coefficient. Combing Eqs. (4) and (5), we can get the nonlinear governing equations, and it is hard to theoretically get the solution of the equations. To obtain the response of the catenary, the improved FEM method is applied to solve above nonlinear equations.

To bear the hydrodynamic force, perpendicularly distributing along the length of catenary, several two-node Euler beam elements are used to represent the catenary. And, we only consider the two translation displacements in $\boldsymbol{x}$-y plane $\left[u_{i}, v_{i}\right]$ and one rotation around $\boldsymbol{z}$ axis $\theta_{i}, \boldsymbol{i}=$ $1,2, \ldots, \boldsymbol{N}+1$, of per node. $\boldsymbol{N}$ is the number of beam elements. Then the dynamic equation is

$(\mathbf{M}+\overline{\mathbf{M}}) \ddot{\mathbf{U}}+\mathbf{C} \dot{\mathbf{U}}+\mathbf{K} \mathbf{U}=\mathbf{P}$

where $\mathbf{M}$ is the structure mass matrix, and $\overline{\mathbf{M}}$ is the added mass matrix. $\mathbf{C}$ is the damping matrix. $\mathbf{K}$ is the stiffness matrix. $\mathbf{P}$ is the hydrodynamic force. $\mathbf{U}$ is the displacement vector. To each beam element, its displacement vector is:

$\mathbf{U}_{i}=\left[u_{i}, v_{i}, \theta_{i}, u_{i+1}, v_{i+1}, \theta_{i+1}\right]^{T} i=1, \cdots, N$

The element mass matrix $\mathbf{M}_{e}$ is:

Table 1

Parameters of the catenary.

\begin{tabular}{llll}
\hline Parameter & Value & Parameter & Value \\
\hline Total length & $7.305 \mathrm{~m}$ & Mass in air & $0.162 \mathrm{~kg} / \mathrm{m}$ \\
Initial horizontal projection & $6.97 \mathrm{~m}$ & Equivalent diameter & $0.0052 \mathrm{~m}$ \\
Initial vertical projection & $1.2 \mathrm{~m}$ & Elastic modulus & $77.2 \mathrm{GPa}$ \\
\hline
\end{tabular}

$\mathbf{M}_{e}=\frac{\rho A l_{e}}{2} \cdot \operatorname{diag}[110110]$

The element stiffness matrix $\mathbf{K}_{e}$ is composed of two parts, i.e. the first part is related to the axial displacement $\left[u_{i}, u_{i+1}\right]$, and the second part is related to the lateral displacement $\left[v_{i}, \theta_{i}, v_{i+1}, \theta_{i+1}\right]$, and it is written as:

$\mathbf{K}_{e}^{1}=\frac{E A}{l_{e}}[1-1-11] \mathbf{K}_{e}^{2}=\frac{E I}{l_{2}^{3}}\left[\begin{array}{cccc}12 & 6 l_{e} & -6 l_{e} & 6 l_{e} \\ 6 l_{e} & 4 l_{e}^{2} & -6 l_{e} & 3 l_{e}^{2} \\ -12 & -6 l_{e} & 12 & -6 l_{e} \\ 6 l_{e} & 3 l_{e}^{2} & -6 l_{e} & 4 l_{e}^{2}\end{array}\right]$

where $l_{e}$ is the element length, based on the matrix in Eq. (9) we can obtain the element stiffness matrix $\mathbf{K}_{e}$. Then all the components in Eq. (6) will be obtained by superimpose all the element matrices.

In our improved FEM model, to consider large rotation and translation motions of the catenary, the rotation DOFs of beam elements is not constrained. Then the rotational DOFs $\theta$ will change into $\theta, \theta^{\prime}$, and the displacement vector of every element, Eq. (7), is rewritten as:

$\mathbf{U}_{i}^{\prime}=\left[u_{i}, v_{i}, \theta_{i}, \theta_{i}^{\prime}, u_{i+1}, v_{i+1}, \theta_{i+1}, \theta_{i+1}^{\prime}\right]^{T} i=2, \cdots, N-1$

It is seen that, because of the additional DOF $\theta^{\prime}$, there exist singularity of the stiffness matrix. To eliminate this singularity, we use additional constraints, i.e. an original shape calculated through traditional static method, see Eq. (1), as the definite condition.

Owing to the increase number of DOFs and strong structural nonlinear characteristics, solving the governing equations is very time consuming. Here, super-element is used for sake of a better efficiency of computation process. The catenary is discrete by a set of super-elements. The processing of each super-element results in a reduced set of matrices (mass, damping, stiffness, and loading) that represent the properties of the catenary. These reduced matrices are assembled in the residual structure, and the assembly solution is performed. Data recovery for each super-element is performed by expanding the solution at the attachment points. A more simplified presentation of the static condensation theory is included here for completeness.

The equilibrium equation of the whole structure is:

$[K] \cdot[U]=[P]$

This equation may be expanded to show the interior DOFs (referred to the $a$-set) and the boundary/exterior DOFs (referred to $O$-set) partitions as:

$\left[\begin{array}{cc}{\left[K_{o o}\right]^{T}} & {\left[K_{o a}\right]} \\ {\left[K_{o a}\right]^{T}} & {\left[K_{a a}\right]}\end{array}\right] \cdot\left[\begin{array}{l}{\left[U_{o}\right]} \\ {\left[U_{a}\right]}\end{array}\right]=\left[\begin{array}{l}{\left[P_{o}\right]} \\ {\left[P_{a}\right]}\end{array}\right]$

where $[K]$ is the stiffness matrix, $[U]$ is the displacement vector and $[P]$ is the load vector. In Eq. (12), the matrix $\left[K_{o o}\right],\left[U_{o}\right]$ and $\left[P_{o}\right]$ represent the reduced stiffness matrix, displacement vector and load vector of the super-elements respectively. The equation can be written as:

$\left[\begin{array}{cc}{\left[K_{o o}^{*}\right]} & {[0]} \\ {\left[K_{o a}^{*}\right]^{T}} & {[I]}\end{array}\right] \cdot\left[\begin{array}{l}{\left[U_{o}\right]} \\ {\left[U_{a}\right]}\end{array}\right]=\left[\begin{array}{c}{\left[P_{o}^{*}\right]} \\ {\left[P_{a}^{*}\right]}\end{array}\right]$

Then we get the governing equation of the super-elements as

$\left[K_{o o}^{*}\right] \cdot\left[U_{o}\right]=\left[P_{o}^{*}\right]$

Obviously, the order of Eq. (14) is significantly reduced comparing with the original equation Eq. (11), thus the computational efficiency could increase. Solving Eq. (14), we can obtain the displacements of the boundary/exterior nodes $\left[U_{o}\right]$. Then, the displacement vector of the interior nodes can be derived by the second formula of Eq. (13). 


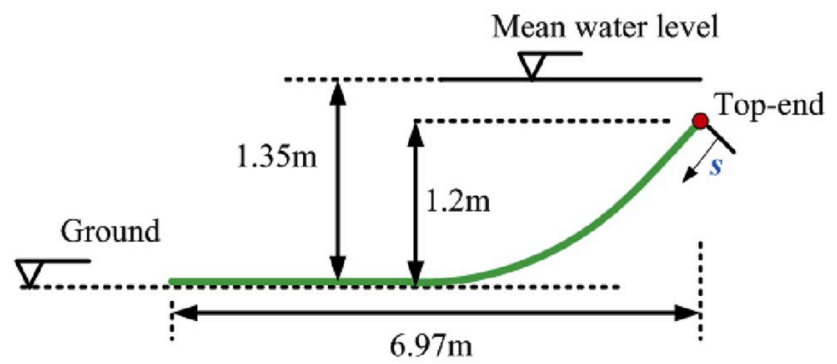

(a) Schematic of the experimental catenary

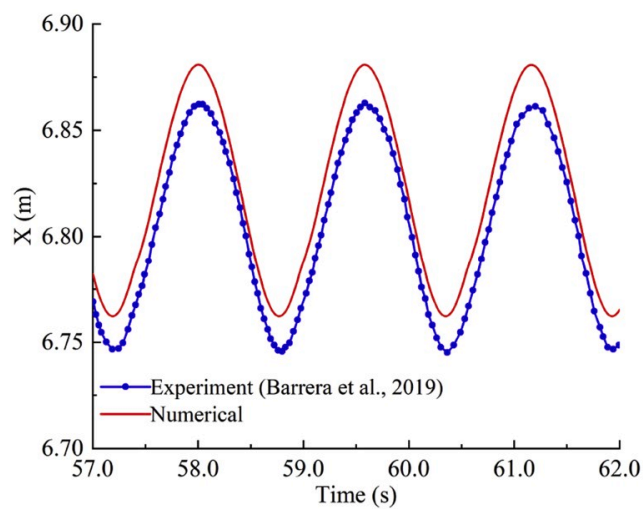

(c) Horizontal displacement of marker $1(\mathrm{~s}=0.2 \mathrm{~m})$

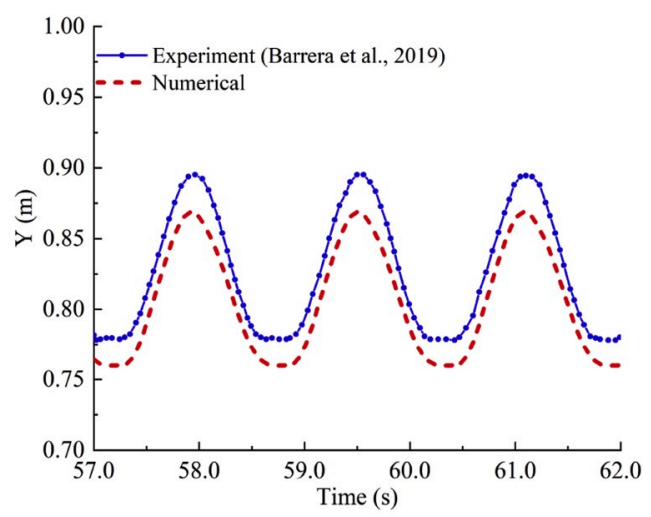

(e) Vertical displacement of marker $3(\mathrm{~s}=0.6 \mathrm{~m})$

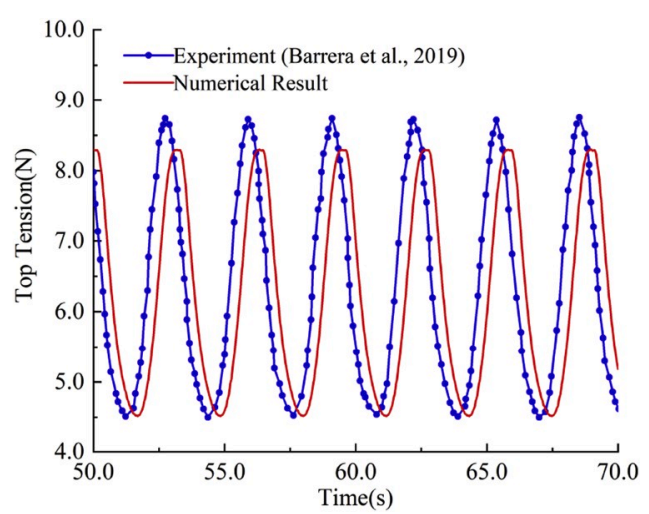

(b) Time history of the top tension

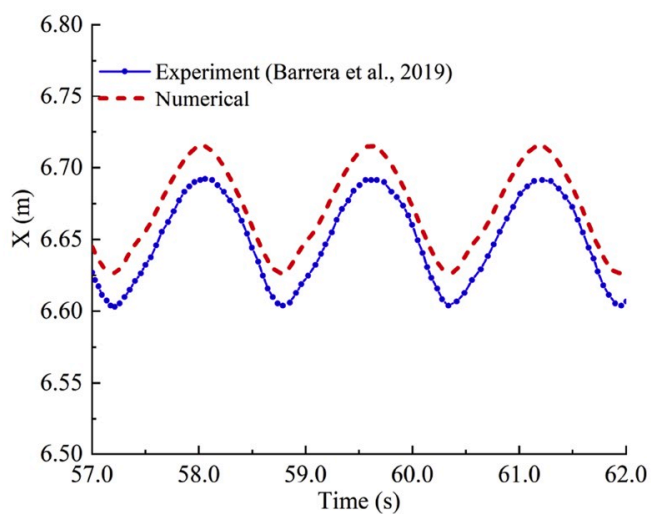

(d) Horizontal displacement of marker $2(\mathrm{~s}=0.4 \mathrm{~m})$

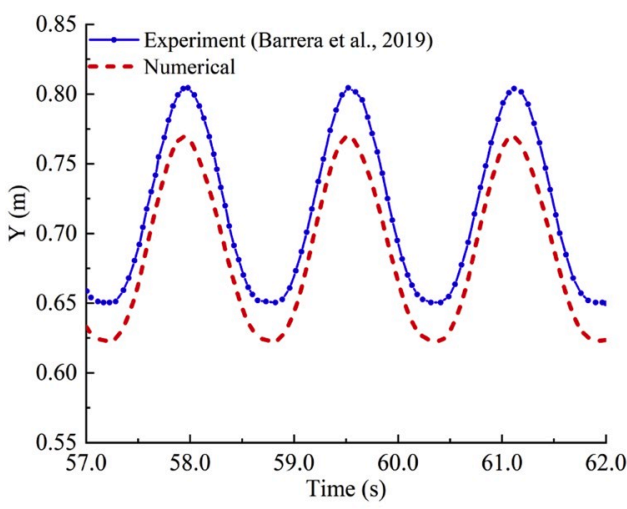

(f) Vertical displacement of marker $4(\mathrm{~s}=0.8 \mathrm{~m})$

Fig. 2. Comparison of numerical and experimental results.

Table 2

Comparison of the numerical and experimental displacement results.

\begin{tabular}{lllll}
\hline Markers & Displacement & Numerical/m & Experimental/m & Error/\% \\
\hline Marker 1 & Maximum & 6.881 & 6.863 & 0.262 \\
& Amplitude & 0.119 & 0.118 & 0.847 \\
Marker 2 & Maximum & 6.715 & 6.691 & 0.358 \\
& Amplitude & 0.089 & 0.087 & 2.298 \\
Marker 3 & Maximum & 0.869 & 0.895 & -2.905 \\
& Amplitude & 0.109 & 0.116 & -6.304 \\
Marker 4 & Maximum & 0.769 & 0.804 & -4.353 \\
& Amplitude & 0.146 & 0.153 & -4.575 \\
\hline
\end{tabular}

\subsection{Model verification}

\subsubsection{Case 1}

To verify our numerical model, the catenary tension and displacement response caused by top-end motion are calculated and compared with the experimental results (Barrera et al., 2019). The parameters of the catenary are listed in Table 1, and Fig. 2a shows a schematic diagram of the structure. Here the top-end surges are considered, of which the surge amplitude is $75 \mathrm{~mm}$ and the surge periods are $3.16 \mathrm{~s}$ and $1.58 \mathrm{~s}$ respectively.

The top tension comparison at 3.16s surge period is shown in Fig. $2 \mathrm{~b}$. It can be seen that the tension values are in good agreement with the experiment, although the phase of time history is slightly different from the experiment. The calculated minimum tension, $6.25 \mathrm{~N}$, is almost 
Table 3

Parameters of the second catenary mooring-line.

\begin{tabular}{|c|c|c|c|}
\hline Parameter & Value & Parameter & Value \\
\hline Total length & $21.0 \mathrm{~m}$ & $\begin{array}{l}\text { Equivalent hydrodynamic } \\
\text { diameter }\end{array}$ & $0.0034 \mathrm{~m}$ \\
\hline $\begin{array}{l}\text { Initial horizontal } \\
\text { projection }\end{array}$ & $19.872 \mathrm{~m}$ & Mass per unit length & $\begin{array}{l}0.069 \mathrm{~kg} / \\
\mathrm{m}\end{array}$ \\
\hline $\begin{array}{l}\text { Initial vertical } \\
\text { projection }\end{array}$ & $5.0 \mathrm{~m}$ & Wet weight per unit length & $\begin{array}{l}0.5872 \mathrm{~N} / \\
\mathrm{m}\end{array}$ \\
\hline Axial stiffness & $3.4 \mathrm{e} 5 \mathrm{~N}$ & & \\
\hline
\end{tabular}

consistent with the experimental value. While the maximum tension, $8.29 \mathrm{~N}$, is slightly smaller than the experimental value of $8.73 \mathrm{~N}$, and the difference is about $5.1 \%$.

In addition to the tension comparison, catenary displacement responses at different positions along catenary length, with the surge period 1.58s, are also compared. In Fig. 2c-f, the numerical and experimental displacements at the 4 markers, i.e. located at $0.2 \mathrm{~m}$ (Marker 1), 0.4m (Marker 2), 0.6m (Marker 3), 0.8m (Marker 4) respectively from the top-end, are presented. It shows that the numerical displacements agree well with the experimental results. And, the horizontal displacement is slightly larger than the experiment (see Fig. $2 \mathrm{c}$ and d), while the vertical displacement is smaller than the experiment (see Fig. 2 e and f). The maximum difference is less than $7 \%$ (see Table 2).

\subsubsection{Case 2}

Further, the comparison between our numerical and the commercial code 3DFloat (José et al., 2017) is also presented. The tension and displacement responses of the catenary caused by top-end motion are calculated. The parameters of the catenary are listed in Table 3, here the top-end surges are considered, of which the surge amplitude is $0.125 \mathrm{~m}$ and the surge period is $1.58 \mathrm{~s}$. The tension and displacement responses of a marker located at $2.646 \mathrm{~m}$ from the top-end are compared in Fig. 3. It can be seen that both the tension and displacement responses agree well with the results of 3DFloat.

\section{Nonlinear restoring performance and dynamic response of catenary mooring-lines}

Here the catenary mooring-line of a 5-MW floating wind turbine is selected as the study object, and its structural and geometrical parameters of the catenary are listed in Table 4. Based on the related researches (Karimirad, 2010; Jeon et al., 2013), the surge amplitude of the floating spar is several meters under regular environment condition. In our study, the amplitude range of the top-end motion is $3-8 \mathrm{~m}$. As for the motion frequency, a wider range was considered, i.e. $0.025 \mathrm{~Hz}-0.2 \mathrm{~Hz}$ compared to the regular wave frequency $0.1 \mathrm{~Hz}$ so as to comprehensively examine the influence of top-end motion on the dynamic behavior of the marine catenary. The restoring performance and its hysteresis of the catenary mooring-line are discussed firstly. Then the dynamic response of the catenary under the motion of top-end float is analyzed based on our numerical simulations, i.e. the displacement, velocity and tension of the catenary along structural span are calculated and compared with the quasi-static results. Sinusoidal surge (horizontal) and heave (vertical) motion with different oscillation frequencies and amplitudes are used as the excitation at top end. Here, Eq. (5) is used to calculate the fluid damping force acting on the catenary, where the values of added mass and damping coefficients are both chosen as 1.0 (Karimirad, 2013). Regarding the fluid damping being much larger than the structural damping, the structural damping is neglected in this study.

\subsection{The non-linear restoring stiffness and its hysteresis}

In fact, because of involvement of dynamic behaviors, i.e. the inertial and damping effect, the restoring stiffness may change, compared with the originally static one. Given that catenary mooring line mainly functions to control the position of floating body and to prevent large surge/sway displacement, the horizontal (surge) restoring stiffness will be examined here under conditions of different top-end amplitudes, i.e. $\mathrm{A}=7,5,4$ and $3 \mathrm{~m}$, and different periods, i.e. $\mathrm{T}=5,10,20,30$ and 40s. And, the selected results are presented in Fig. 4 and Fig. 5.

Fig. 4a shows the restoring stiffness curve of the catenary, at $10 \mathrm{~s}$ period and $4 \mathrm{~m}$ amplitude, and also the static curve as a comparison. Interestingly, the dynamic stiffness curve shows that the top tension is no longer linearly related to only the top-end displacement as it does for case of quasi-static scenario, but, notably, it depends on both the top-end displacement and velocity approximately in a way of approximately ellipse loop, which is called hysteresis loop. Or, the dynamic restoring force actually has a directional property mainly owing to the involvement of the dynamic behavior which principally depends on both the

Table 4

The geometrical and material parameters of the catenary.

\begin{tabular}{lcll}
\hline Geometrical & Value & Geometrical & Value \\
\hline Length & $800 \mathrm{~m}$ & Young's modulus & $210 \mathrm{GPa}$ \\
Initial horizontal projection & $706 \mathrm{~m}$ & Equivalent Mass density & $71.2 \mathrm{~kg} / \mathrm{m}$ \\
Initial vertical projection & $350 \mathrm{~m}$ & Poisson's ratio & 0.3 \\
Diameter & $0.19 \mathrm{~m}$ & & \\
\hline
\end{tabular}

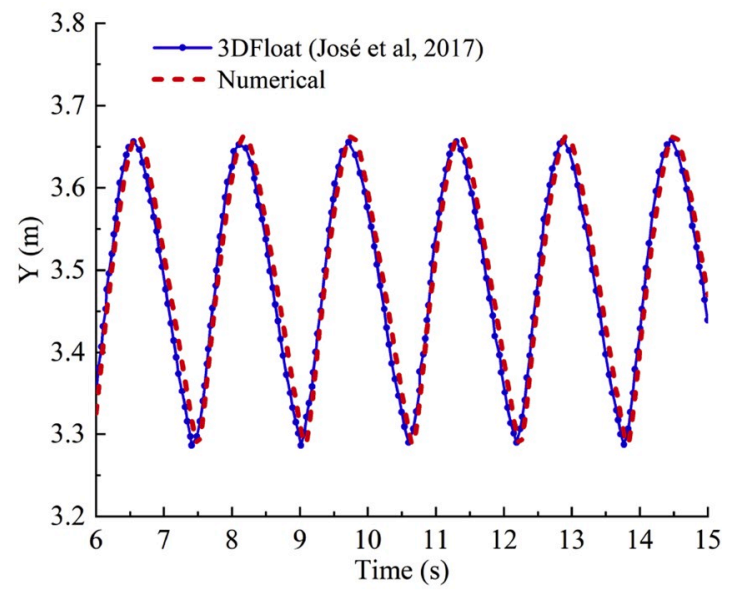

(b) Vertical displacement

(a) Time history of the top tension

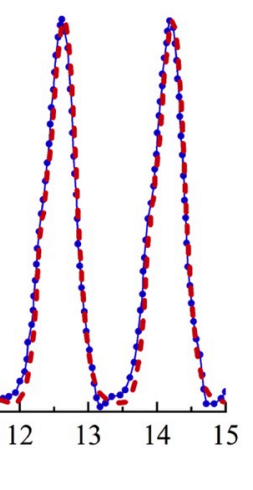

Fig. 3. Comparison of numerical and results provided by 3DFloat. 


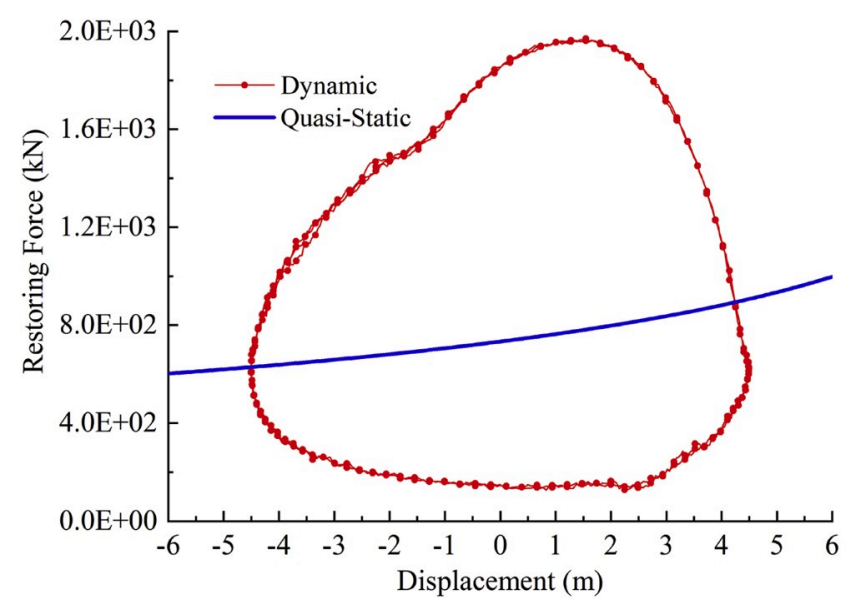

(a) Restoring stiffness of the mooring line

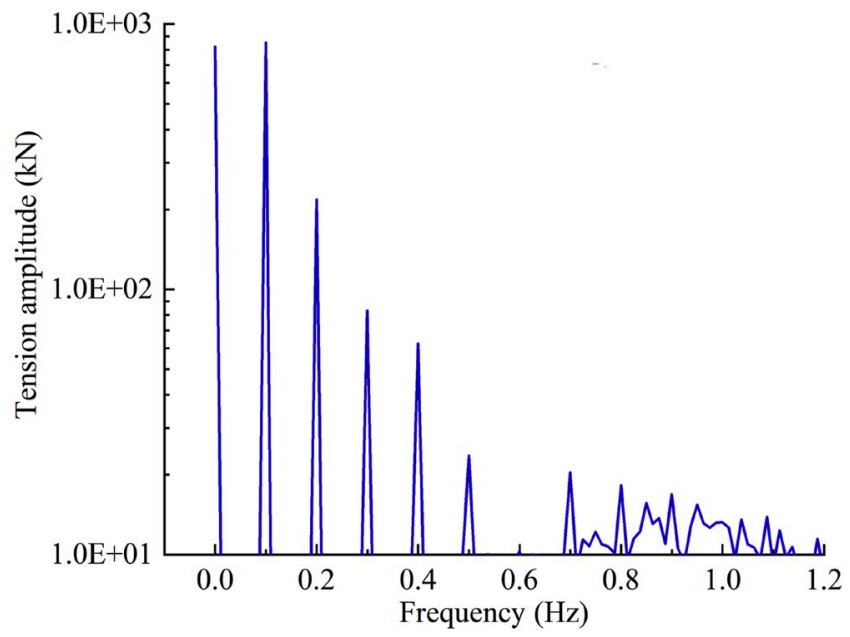

(b) Spectrum of the restoring force

Fig. 4. Restoring performance of the single mooring line.

amplitude and direction of the top-end motion. And differently from the static stiffness, the dynamic restoring force does not get its maximum value at the maximum displacement but at a smaller displacement, that produces a bigger dynamic stiffness. The hysteresis character of the dynamic stiffness is mainly due to the damping effect coming from the structure and fluid of the mooring-line. The spectrum plot, shown in Fig. 4b, indicates that the peak values at frequencies of odd times of the excitation frequency is much larger than others.

In addition, if under the same loop area $A_{d}$, Fig. 5 presents two groups of hysteresis loops respectively with the loop area $A_{d}=8000 \mathrm{kNm}$ and $A_{d}=12000 \mathrm{kNm}$. It is noted that the hysteresis loop may shape from horizontal ellipse to more vertical one as the period (or amplitude) decreases.

\subsection{Hysteretic behavior at various frequencies and amplitudes}

The loop area of the stiffness curve under different top-end surge conditions is shown in Fig. 6. It shows that the loop area of the stiffness curve get larger as the amplitude (and/or the frequency) increases. In other words, the hysteresis behavior of the dynamic stiffness gets more obvious as the amplitude and/or frequency increase.

For simplicity and representativeness, here a mass-spring damping system with on DOF is taken as an example to illustrate relationship between the energy consumption and the amplitude/frequency of the system. Regarding that the damping effect coming from fluid dynamics is much larger that the structural dynamics, we assume the damping

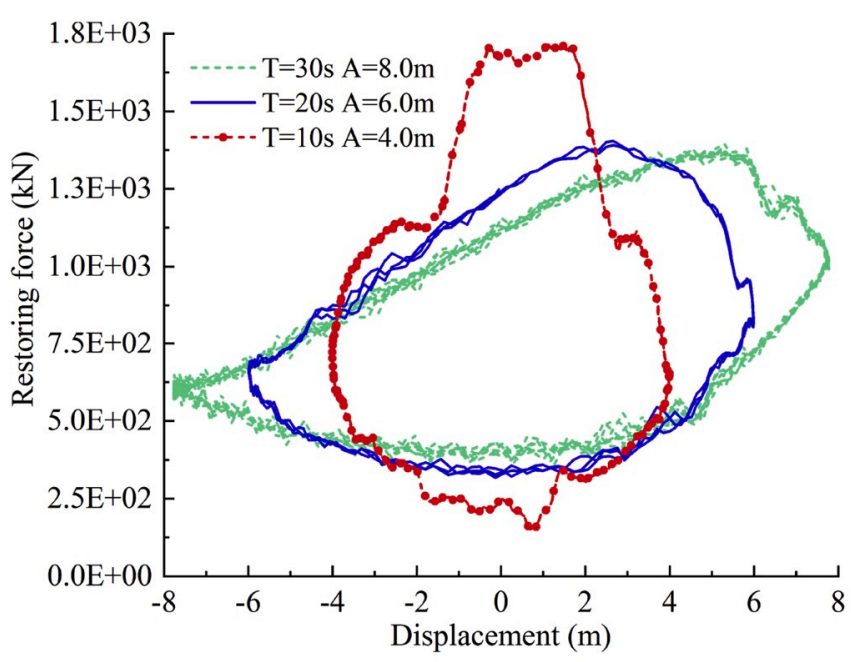

(a) $A_{d}=8000$

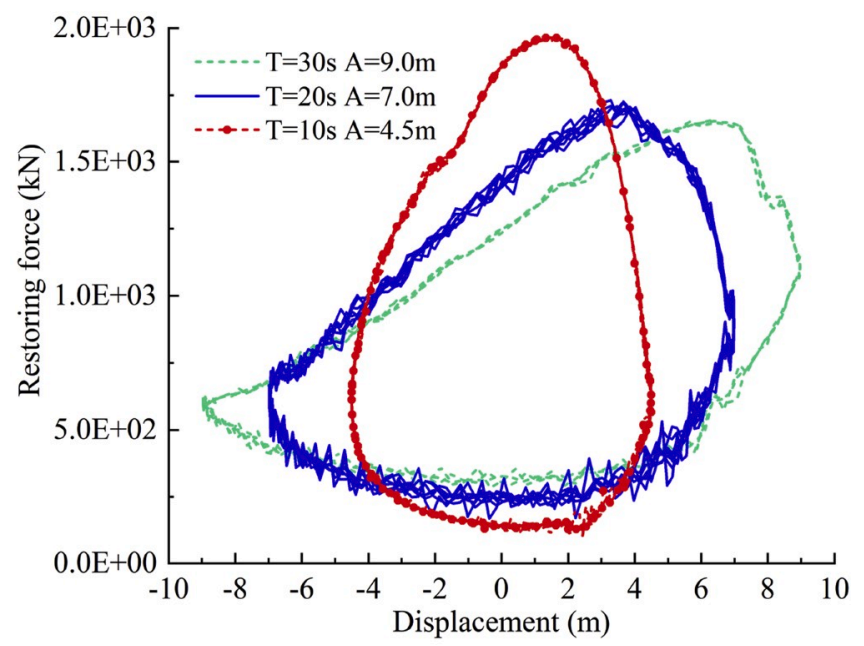

(b) $A_{d}=12000$

Fig. 5. Hysteresis loop shapes changing with the frequency and amplitude.

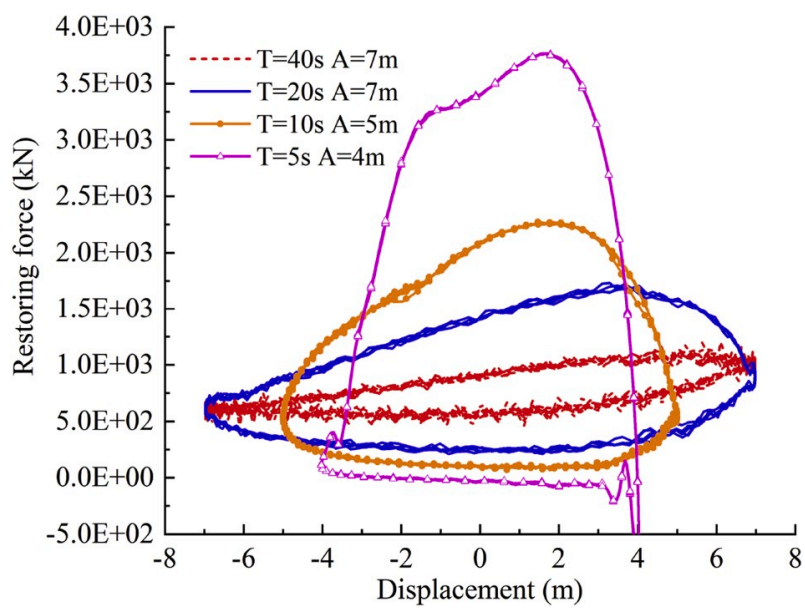

Fig. 6. Restoring loops at different frequency and amplitude. 


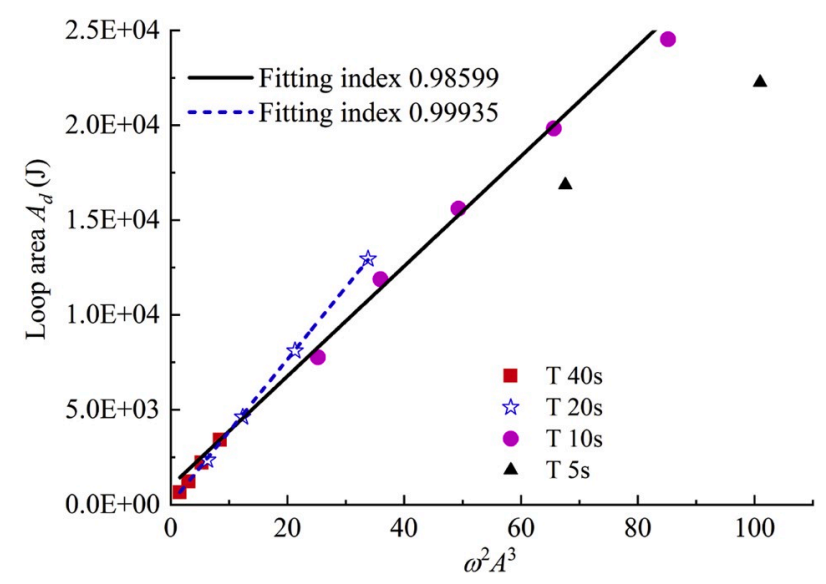

Fig. 7. Loop's area under different conditions.

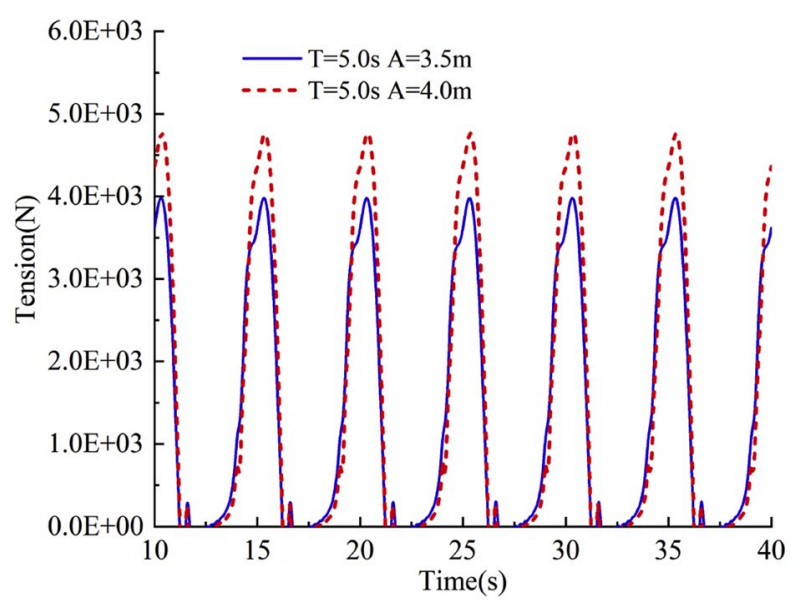

(a) Time history of the catenary top tension

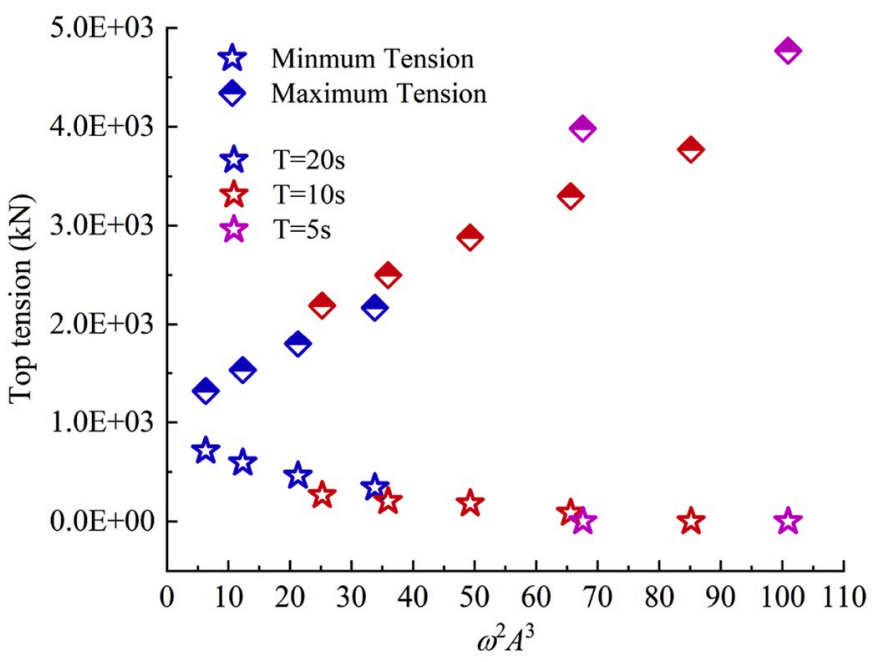

(b) Top tension under different conditions

Fig. 8. Top tension response of the catenary.

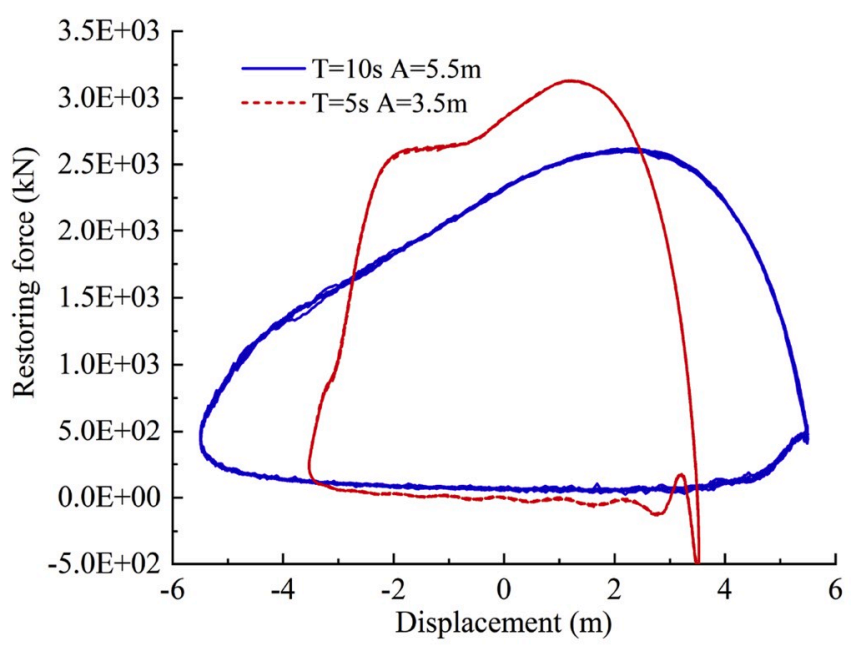

(a) $\omega_{d}^{2} A_{0}^{3}=65$

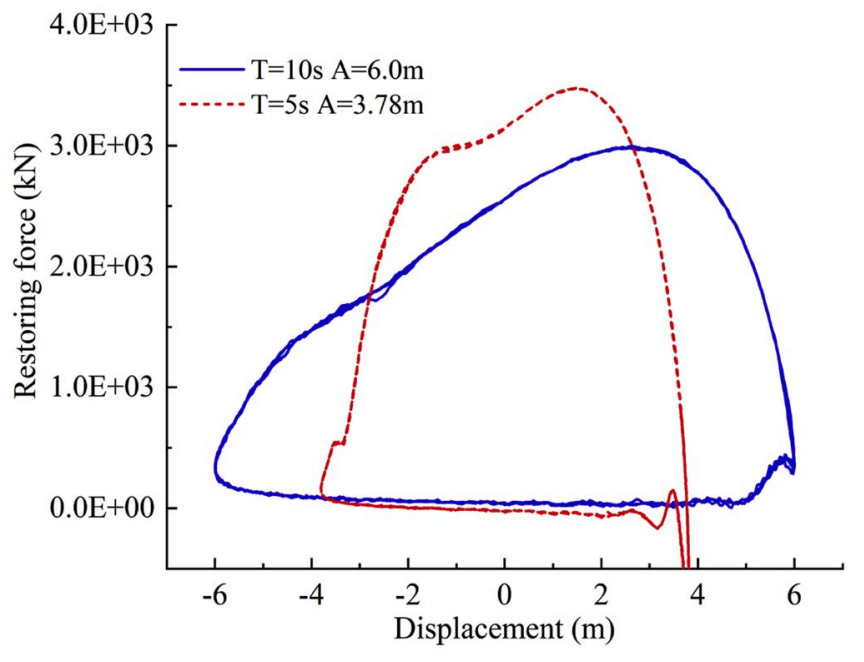

(b) $\omega_{d}^{2} A_{0}^{3}=85$

Fig. 9. Comparison of the restoring performance when slack happens.

force is $F_{d}=c_{0} \dot{y}^{2}$, where $y=A_{0} \sin \left(\omega_{d} t\right)$ is the displacement and $c_{0}$ is the damping coefficient. Then the energy consumption of one period is the integration written as follows (Thomson and Dahleh, 2002):

$$
\begin{aligned}
W_{d} & =4 \int_{0}^{y_{\max }} F_{d} d y=4 \int_{0}^{\frac{\pi}{2 \omega_{d}}} c_{0} \omega_{d}^{2} A_{0}^{3}\left(1-\sin ^{2}\left(\omega_{d} t\right)\right) d t\left(\sin \left(\omega_{d} t\right)\right) \\
& =\frac{8}{3} c_{0} \omega_{d}^{2} A_{0}^{3}
\end{aligned}
$$

In other words, the loop area $A_{d}$ represents the energy consumption during one motion period, and it is proportional to the quadratic frequency $\left(\omega_{d}^{2}\right)$ and cubic amplitude $A_{0}^{3}$. The plot of loop area $A_{d}$ versus $\omega_{d}^{2} A_{0}^{3}$ is presented in Fig. 7. It shows that $A_{d}$ approximately rises linearly with $\omega_{d}^{2} A_{0}^{3}$, and the fitting index of the linear fitting function is about 0.986 . So we may say the damping effect of the catenary dynamics is mainly responsible for the hysteresis of the restoring performance.

It should be pointed out that the results of $\mathrm{T}=5 \mathrm{~s}$ are not included during the linear fitting process. The reason is that in those situations the snaps happen as the value $\omega_{d}^{2} A_{0}^{3}$ gets larger, which may introduce strong nonlinearity of restoring stiffness. Or the deviations of the three results of $\mathrm{T}=5 \mathrm{~s}$ (the squares) from the fitting line are larger and should not be included due to the snap phenomenon and its strong nonlinearity. The happening of slack could be further proven by observing the time history 


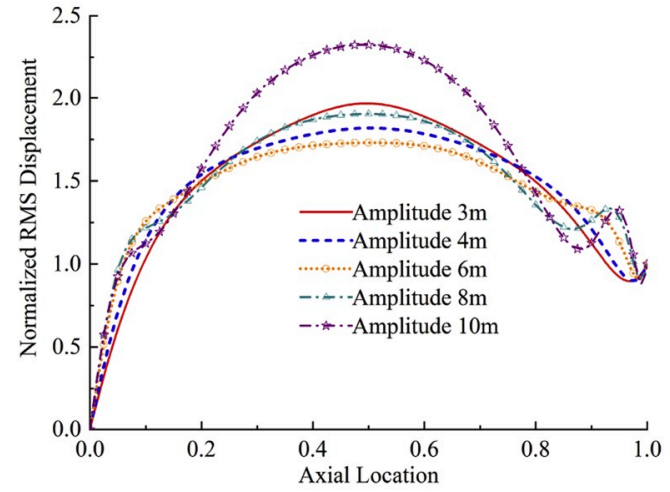

(a) The top-end surge amplitude ranging from $3 \mathrm{~m}$ to $10 \mathrm{~m}$

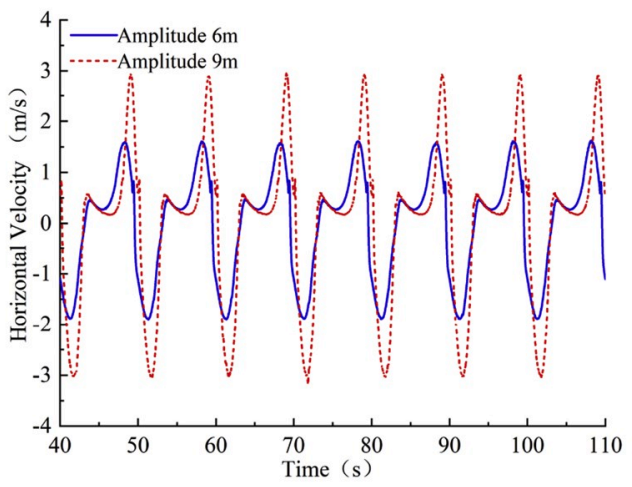

(c) The horizontal velocity

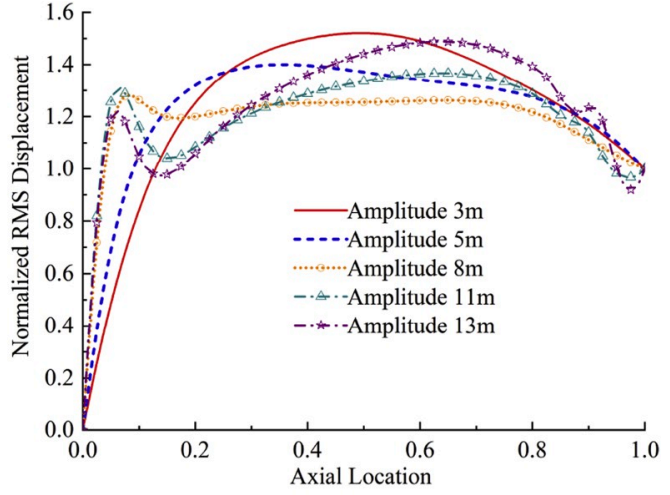

(b) The top-end heave amplitude range from $3-13 \mathrm{~m}$

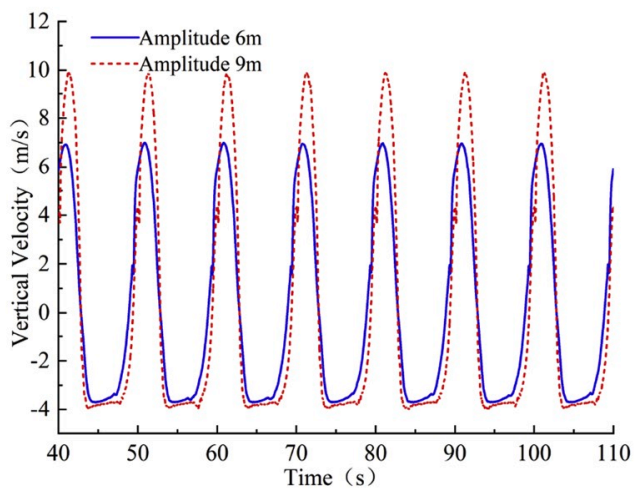

(d) The vertical velocity

Fig. 10. Normalized RMS displacement and velocity response of the middle point.

of the top tension as $\mathrm{T}=5 \mathrm{~s}$, shown in Fig. $8 \mathrm{a}$, where the minimum top tension is approaching zero and an obvious rise of the maximum top tension is seen. Further, the maximum/minimum restoring forces, with the same value of $\omega_{d}^{2} A_{0}^{3}=65$, is compared in Fig. 8b. It shows that as the top-end period gets smaller, it becomes easier for snap to happen (Guo et al., 2017), and, consequently, the maximum tension gets larger. Because the top tension is approaching to zero (and the consequent zero stiffness), there exists a certain time duration when the restoring force does not consume any energy. Then, the energy consumption gets smaller for this case, as shown in Fig. 7. If we include just the data of $\mathrm{T}=$ 40 s and $\mathrm{T}=20$ s, or no snap occurrence, the fitting index in Fig. 7 is
0.999 which is pretty close to the perfect value of 1.0 . That means a better fitting linearity.

To find the reason why the loop area will decrease when slack happens to the catenary, the comparison of the restoring loops with the same value $\omega_{d}^{2} A_{0}^{3}=65,85$ (in the snap/slack region) is shown in Fig. 9. It can be seen that as slack happens (the red loops), the displacement of the top-end becomes somewhat smaller during the time period when the restoring force drops from the maximum point to the minimum. That is to say the energy consumption during this period of time gets smaller. It should be note that there are minus tensions when the top-end motion period is $5 \mathrm{~s}$. The negative restoring force indicates that the catenary

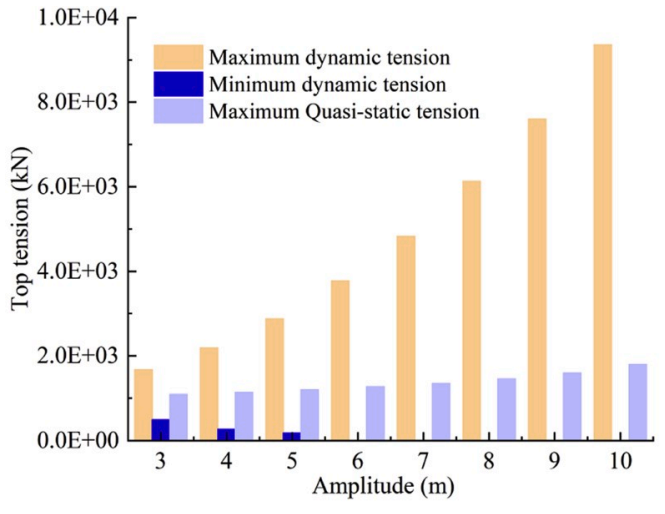

(a) Tension versus top-end amplitude at $0.10 \mathrm{~Hz}$ frequency

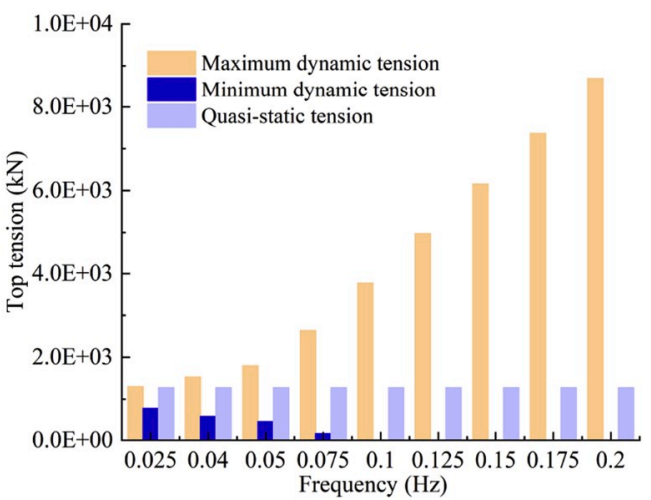

(b) Tension versus top-end frequency at $6 \mathrm{~m}$ amplitude

Fig. 11. Comparison of the maximum tension with the static at various top-end amplitudes/frequencies. 
mooring line is in a slack situation, and the catenary may be compressed for a short time caused by large top-end motion.

\subsection{Impacts of the dynamic behavior on catenary response}

As shown above, with the mooring-line dynamics under consideration, the mooring-line restoring performance has an obvious difference from the quasi-static. That is to say, we should include the dynamic effects of the catenary. Here the dynamic response of the catenary under different top-end motion conditions are examined and compared with the quasi-static.

Fig. 10a shows the RMS (Root-Mean-Square) displacements under the top-end surge with $0.1 \mathrm{~Hz}$ frequency and the amplitudes ranging from $3 \mathrm{~m}$ to $10 \mathrm{~m}$, and Fig. 10b shows the RMS displacement under topend heave (with $0.1 \mathrm{~Hz}$ frequency and $3-13 \mathrm{~m}$ amplitudes). For case of surge motion (see Fig. 10a), the maximum value of catenary displacement occurs almost at the center position along the catenary length. While for case of heave motion (see Fig. 10b), the position of the maximum displacement moves along the catenary length. And, unlike the static case, the maximum displacement does not gradually rise as the top-end amplitude increases, but, instead, and it decreases originally and then increases with the increase of top-end amplitude. It is worthy to note that some shorter waves happen at the catenary ends, and this shorter wave gets more obvious, which consequently can cause higher values of structural local curvature and stress.

If concerning the velocity response, as shown in Fig. $10 \mathrm{c}$ and $\mathrm{d}$, the time histories of the center position along the catenary, with respectively $6 \mathrm{~m}$ and $9 \mathrm{~m}$ top-end surge amplitude, show that the velocity increases with the increase of top-end amplitude. Moreover, slack may happen as the amplitude increases to a certain value, e.g. at $9 \mathrm{~m}$ amplitude (in Fig. 10d) the vertical velocity almost keeps as a constant when it approaches to the minimum value and stay there for a period of time.

The maximum top tensions are compared with the static values in Fig. 11 at different amplitudes and frequencies of top-end motion. The difference between the dynamic and static tensions gets more obvious as the top-end amplitude/frequency increases. Particularly, the dynamic tension is around 5.2 times (in Fig. 11b) of the quasi-static one when slack happens. This slack can be proved by the minimum tension which gradually drops and approaches to zero when the amplitude/frequency exceeds $6 \mathrm{~m} / 0.10 \mathrm{~Hz}$.

\section{Conclusion}

The restoring performance and the hysteresis behavior of the catenary mooring-line are presented and deeply discussed based on the numerical results using our improved FEM model. And the impacts of mooring-line dynamics on the catenary response, including the displacement and tension, are studied while the dynamic results are also compared to the quasi-static. Based our numerical results we have remarks as follows:

1) Owing to the damping effect coming from the fluid and structural dynamics, the mooring-lines stiffness presents a hysteresis behavior which becomes more obvious as the top-end frequency/amplitude gets larger.

2) It is found that the area of hysteresis loop, which indicates the energy consumption during one period, is approximately proportional to $\omega_{d}^{2} A_{0}^{3}$. So we may say the damping effect of the catenary dynamics is mainly responsible for the hysteresis of the restoring performance.

3) The catenary dynamics has a significant effect on the tension (restoring stiffness) and displacement response of the catenary. Particularly, as the mooring-line becomes slack, the maximum tension amplitude is up to 5.2 times, i.e. as the amplitude and frequency are respectively $6 \mathrm{~m}$ and $0.15 \mathrm{~Hz}$, of the quasi-static method.

\section{Declaration of competing interest}

The authors declare there is no conflicts of interest regarding the publication of this paper.

\section{CRediT authorship contribution statement}

Yilun Li: Methodology, Investigation, Writing - original draft. Shuangxi Guo: Validation, Formal analysis, Visualization, Writing original draft. Weimin Chen: Conceptualization, Writing - review \& editing, Supervision. Dingbang Yan: Writing - review \& editing. Jixiang Song: Writing - review \& editing.

\section{Acknowledgments}

The authors of this paper would like to thank the financial supports provided by the Strategic Priority Research Programme of CAS (Grant No. XDA22000000) and the National Natural Science Foundation of China (Grant No. 11372320).

\section{References}

Azcona, J., Munduate, X., González, L., et al., 2017. Experimental validation of a dynamic mooring lines code with tension and motion measurements of a submerged chain. Ocean. Eng. 129, 415-427.

Barrera, C., Guanche, R., Losada, I.J., 2019. Experimental modelling of mooring systems for floating marine energy concepts. Mar. Struct. 63, 153-180.

Chen, X., 2002. Studies on Dynamic Interaction between Deep-Water Floating Structures and Their Mooring/tendon Systems. Texas A\&M University.

Chen, X., Zhang, J., Ma, W., 2001. On dynamic coupling effects between a spar and its mooring lines. Ocean. Eng. 28 (7), 863-887.

Fan, T., Qiao, D., Yan, J., et al., 2017. An improved quasi-static model for mooringinduced damping estimation using in the truncation design of mooring system. Ocean. Eng. 136, 322-329.

Garrett, D.L., 1982. Dynamic analysis of slender rods. J. Energy Resour. Technol. 104 (4), 302-306.

Gobat, J.I., Grosenbaugh, M.A., 2001. A simple model for heave-induced dynamic tension in catenary moorings. Appl. Ocean Res. 23 (3), 159-174.

Guo, S., Chen, W., Fu, Y., 2016. Non-linearly restoring performance of SFT's catenary mooring-lines under consideration of its dynamic behaviors. Procedia Engineering $166,202-211$.

Guo, S., Li, Y., Chen, W., et al., 2017. Non-linearly Restoring Performance of Catenary Mooring-Line under Consideration of its Dynamic Behaviors, ASME 2017, International Conference on Ocean. Offshore and Arctic Engineering. V07AT06A021.

Hsu, W.T., Thiagarajan, K.P., Manuel, L., 2017. Extreme mooring tensions due to snap loads on a floating offshore wind turbine system. Mar. Struct. 55, 182-199.

Huang, S., Vassalos, D., 1993. A numerical method for predicting snap loading of marine cables. Appl. Ocean Res. 15 (4), 235-242.

Jeon, S.H., Cho, Y.U., Seo, M.W., et al., 2013. Dynamic response of floating substructure of spar-type offshore wind turbine with catenary mooring cables[J]. Ocean. Eng. 72, 356-364.

José, A., Xabier, M., Leo, G., et al., 2017. Experimental validation of a dynamic mooring lines code with tension and motion measurements of a submerged chain. Ocean. Eng. 129, 415-427.

Karimirad, M., 2010. Dynamic response of floating wind turbine[J]. Sci. Iran. 17 (2), $146-156$.

Karimirad, M., 2013. Modeling aspects of a floating wind turbine for coupled wave-windinduced dynamic analyses. Renew. Energy 53, 299-305.

Kwan, C.T., Bruen, F.J., 1991. Mooring line dynamics: comparison of time domain, frequency domain, quasi-static analyses. In: Proceeding of the 23rd Offshore Technology Conference: Houston TX, pp. 95-108.

Lindahl, J., Sjoberg, A., 1983. Dynamic analysis of mooring cables. In: Proceedings of Second International Symposium on Ocean Engineering and Ship Handling, Sweden, pp. 281-319.

Love, A.E.H., 2013. A Treatise on the Mathematical Theory of Elasticity. Cambridge University Press.

Mansour, A., Mekki, O.B., Montassar, S., et al., 2018. Catenary-induced geometric nonlinearity effects on cable linear vibrations. J. Sound Vib. 413, 332-353.

Mavrakos, S.A., Chatjigeorgiou, J., 1997. Dynamic behaviour of deep water mooring lines with submerged buoys. Comput. Struct. 64 (1), 819-835.

Musial, W., Butterfield, S., Boone, A., 2004. Feasibility of floating platform systems for wind turbines. In: 23rd ASME Wind Energy Symposium, Reno, NV.

Ogawa, Yoji, 1984. Fundamental analysis of deep sea mooring line in static equilibrium. Appl. Ocean Res. 6 (3), 140-147.

Papazoglou, V.J., Mavrakos, S.A., Triantafyllou, M.S., 1990. Non-linear cable response and model testing in water. J. Sound Vib. 140 (1), 103-115. 
Paulling, J.R., Webster, W.C., 1986. A consistent large-amplitude analysis of the couple response of a TLP and tendon system. In: Proceedings of Offshore Mechanics and Arctic Engineering, vol. 3. OMAE, Tokyo, Japan, pp. 126-133.

Sarkar, A., Eatock Taylor, R., 2002. Dynamics of mooring cables in random seas. J. Fluid Struct. 16 (2), 193-212.

Thomson, W.T., Dahleh, M.D., 2002. Theory of Vibration with Application, third ed. CBS Publishers, New Delhi.

Vassalos, D., Huang, S., 1996. Dynamics of small-sagged slack-taut marine cables. Comput. Struct. 58 (3), 557-562.

Xiong, L., Yang, J., Zhao, W., 2016. Dynamics of a taut mooring line accounting for the embedded anchor chains. Ocean. Eng. 121, 403-413.
Yong-Pyo, Hong, et al., 2005. An experimental study on the extreme motion responses of a spar platform in the heave resonant waves. In: The Fifteenth International Offshore and Polar Engineering Conference. International Society of Offshore and Polar Engineers.

Zhang, S., Tang, Y., Liu, X., 2010. Analysis of nonlinear dynamic response of mooring lines in deepwater. In: ASME 2010 29th International Conference on Ocean, Offshore and Arctic Engineering. American Society of Mechanical Engineers, pp. 489-494.

Zhang, S., Tang, Y., Liu, X., 2012. Experimental investigation of nonlinear dynamic tension in mooring lines. J. Mar. Sci. Technol. 17 (2), 181-186. 